\title{
OPEN
}
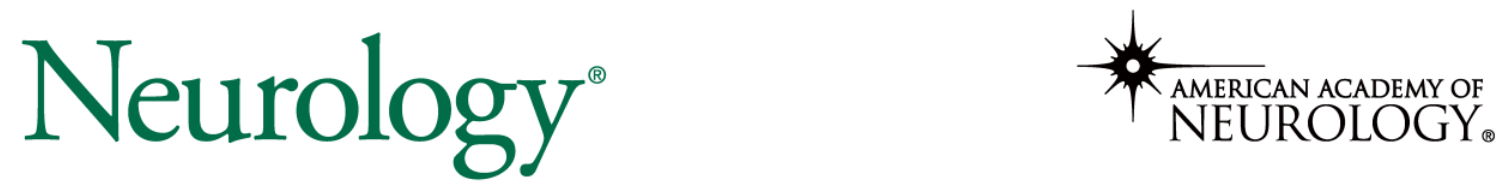

The most widely read and highly cited peer-reviewed neurology journal

The Official Journal of the American Academy of Neurology

Neurology Publish Ahead of Print

DOI: 10.1212/WNL.0000000000200005

\section{Impact of Small Vessel Disease Progression on Long-term Cognitive and Functional}

\section{Changes After Stroke}

This is an open access article distributed under the terms of the Creative Commons Attribution

License 4.0 (CC BY), which permits unrestricted use, distribution, and reproduction in any medium, provided the original work is properly cited.

Neurology® Published Ahead of Print articles have been peer reviewed and accepted for publication. This manuscript will be published in its final form after copyediting, page composition, and review of proofs. Errors that could affect the content may be corrected during these processes. 


\section{Author(s):}

Una Clancy, MB BAO BCh ${ }^{1}$; Stephen DJ Makin, $\mathrm{PhD}^{1,2}$; Caroline A McHutchison, $\mathrm{PhD}^{1}$; Vera Cvoro, $\mathrm{MD}^{1}$; Francesca M Chappell, $\mathrm{PhD}^{1}$; Maria del C. Valdés Hernández, $\mathrm{PhD}^{1}$; Eleni Sakka, MSc ${ }^{1}$; Fergus Doubal, $\mathrm{PhD}^{1}$; Joanna M. Wardlaw ${ }^{1}$

\section{Corresponding Author:}

Joanna M. Wardlaw

joanna.wardlaw@ed.ac.uk

Affiliation Information for All Authors: 1. Centre for Clinical Brain Sciences, Edinburgh Imaging and the UK Dementia Research Institute, University of Edinburgh, United Kingdom2. Centre For Rural Health, Institute of Applied Health Sciences, University of Aberdeen, United Kingdom

\section{Contributions:}

Una Clancy: Drafting/revision of the manuscript for content, including medical writing for content; Analysis or interpretation of data

Stephen DJ Makin: Drafting/revision of the manuscript for content, including medical writing for content; Major role in the acquisition of data; Analysis or interpretation of data

Caroline A McHutchison: Drafting/revision of the manuscript for content, including medical writing for content; Major role in the acquisition of data; Analysis or interpretation of data

Vera Cvoro: Drafting/revision of the manuscript for content, including medical writing for content; Major role in the acquisition of data; Analysis or interpretation of data

Francesca M Chappell: Drafting/revision of the manuscript for content, including medical writing for content; Analysis or interpretation of data 
Maria del C. Valdés Hernández: Drafting/revision of the manuscript for content, including medical writing for content; Major role in the acquisition of data

Eleni Sakka: Drafting/revision of the manuscript for content, including medical writing for content; Major role in the acquisition of data

Fergus Doubal: Drafting/revision of the manuscript for content, including medical writing for content Joanna M. Wardlaw:

Figures: 4

Tables: 2

Search Terms: [ 2 ] All Cerebrovascular disease/Stroke, [ 25 ] All Cognitive Disorders/Dementia, [ 120 ] MRI

Study Funding: This study was supported in part by the Wellcome Trust (WT088134/Z/09/A; funded most of the data collection and S.D.J.M). For the purpose of open access, the author has applied a CC BY public copyright licence to any Author Accepted Manuscript version arising from this submission. The three year follow-up was also funded by Chest, Heart Stroke Scotland:Res14/A157 (CAM); support for the research was also received from: NHS Research Scotland (VC, FD); Row Fogo Charitable Trust Centre for Research into Aging and the Brain (BROD.FID3668413; M.d.C.V.H., ES); the European Union Horizon 2020 project 666881, SVDs@Target (F.M.C.); Scottish Funding Council, Scottish Imaging Network, A Platform for Scientific Excellence Initiative; Chief Scientist Office Scotland (UC;CAF/18/08); Stroke Association Princess Margaret Research Development Fellowship (UC); Stroke Association-Garfield Weston Foundation Senior Clinical Lectureship (FND;TSALECT 2015/04). Funding from Fondation Leducq (16-CVD-05) and UK Dementia Research Institute (JMW), funded by the UK Medical Research Council, Alzheimer's Society and Alzheimer's Research UK, is gratefully acknowledged.

Disclosures: U. Clancy, S.D.J. Makin, F.M. Chappell, M.dC. Valdés Hernández, F. Doubal, V. Cvoro, C.A. McHutchison, E. Sakka, and J.M. Wardlaw hold grants from government or charitable agencies that funded the research but have no other disclosures. 


\section{Abstract}

\section{Background and Objectives}

The severity of white matter hyperintensities (WMH) at presentation with stroke associates with post-stroke dementia and dependency. However, WMH can decrease or increase after stroke, prediction of cognitive decline is imprecise, and there are few data assessing longitudinal interrelationships between changing $\mathrm{WMH}$, cognition, and function after stroke, despite the clinical importance.

\section{Methods}

We recruited patients within three months of a minor ischaemic stroke, defined as NIHSS score $<8$ and not expected to result in a modified Rankin Score (mRS) $>2$. Participants repeated $\mathrm{MRI}$ at one year, and cognitive and $\mathrm{mRS}$ assessments at one and three years. We ran longitudinal mixed-effects models assessing change in Addenbrooke's Cognitive Examination-Revised (ACE-R) and modified Rankin Scores (mRS). For mRS, we assessed longitudinal WMH volumes (cube root; percentage intracranial volume [ICV]), adjusting for age, NIHSS, ACE-R, stroke subtype, and time to assessment. For ACE-R, we additionally adjusted for ICV, $\mathrm{mRS}$, premorbid IQ, and vascular risk factors. We then used a multivariate model to jointly assess changing cognition/mRS, adjusted for prognostic variables, using all available data.

\section{Results}

We recruited 264 patients; mean age was 66.9 (SD 11.8); 41.7\% were female; median mRS 1 (IQR 1-2). One year after stroke, normalised WMH volumes associated more strongly with one-year ACE-R ( $\beta=-0.259,95 \%$ confidence interval 
$[\mathrm{Cl}]-0.407$ to -0.111 more $\mathrm{WMH}$ per 1 -point ACE-R decrease, $p=0.001$ ) compared to subacute $\mathrm{WMH}$ volumes and ACE-R $(\beta=0.105,95 \% \mathrm{Cl}-0.265$ to $0.054, p=0.195)$. Three-year mRS associated with three-year ACE-R $(\beta=-0.272,95 \% \mathrm{Cl}-0.429$ to $0.115, p=0.001)$. Combined change in baseline-one-year jointly assessed ACE$\mathrm{R} / \mathrm{mRS}$ associated with fluctuating WMH volumes ( $F=9.3, p=0.03)$.

\section{Discussion}

After stroke, fluctuating WMH mean that one-year, but not baseline, WMH volumes associate strongly with contemporaneous cognitive scores. Co-varying longitudinal decline in cognition and independence post-stroke, central to dementia diagnosis, is associated with increasing $\mathrm{WMH}$ volumes.

\section{Introduction}

Cerebral small vessel disease (SVD) is common in patients with stroke and is a common cause of stroke and vascular dementia. ${ }^{1}$ White matter hyperintensities $(\mathrm{WMH})$, a key feature of SVD, have been shown to decrease as well as increase in stroke ${ }^{2}$ cognitively impaired ${ }^{3,4}$ older community-dwelling adults investigated for neurological or cognitive symptoms, ${ }^{5}$ and healthy adults (mean age 63 ).${ }^{6}$ It is not clear how the timing of SVD lesion change relates to the clinical development of stroke and dementia.

To diagnose dementia, evidence of coexisting decline in both cognitive and functional ability is required. After stroke, we usually define functional status according to the degree of post-stroke disability measured by the modified Rankin Scale (mRS), i.e. the level of independence in carrying out activities of daily living. In particular, 'mild neurocognitive disorder' is distinguished from 'major neurocognitive disorder' by the presence of increasing dependence. ${ }^{7,8}$ This is particularly relevant after stroke, as 
both worsening $\mathrm{mRS}$ and cognitive impairment are common and interrelated three years after minor stroke. ${ }^{9}$ However, it is not known whether SVD progression might influence the interaction of these impairments.

In cross-sectional studies, worse white matter hyperintensities (WMH) at stroke presentation are associated with worse cognition assessed concurrently as well as long term..$^{10,11}$ However, it is less clear whether longitudinal WMH change predicts coexistent post-stroke cognitive or functional impairment. Relatively small MRI studies assessing WMH progression and post-stroke cognitive decline have not detected a longitudinal association, however follow-up sample sizes may have been underpowered, ranging from $n=30$ to $n=94$ participants ${ }^{12-17}$ (systematic search for relevant papers and summary of their characteristics are described in eTable 1 and eTable 2 in the Supplement). Additionally, we and others showed recently that WMH can decrease as well as increase long term ${ }^{2,5}$ and, although the reasons are poorly understood, fluctuations in WMH have not been accounted for in longitudinal studies. Although numerous studies have assessed baseline WMH and mRS change after stroke, ${ }^{10}$ we are not aware of any studies that have assessed WMH progression and mRS change after stroke (eTable 1, eTable 2 in the Supplement), or if WMH progression or severity at specific time-points affects variation between both mRS and cognition post-stroke, despite these measures being collected increasingly by ongoing studies, ${ }^{18,19}$

The present in-depth analysis builds on previous work from the Mild Stroke Study- ${ }^{9}$ on one- and three-year outcomes after stroke which included predictors of cognition and cognition-mRS relationships, ${ }^{9}$ but did not assess longitudinal change incorporating all three elements of cognition, $\mathrm{mRS}$ and $\mathrm{WMH}$. 
We aimed to determine whether WMH associations with cognition and mRS vary at different time-points post-stroke; whether associations vary according to intraindividual trajectories; whether longitudinal WMH volume change associates with change in cognition and mRS as a co-primary, co-varying endpoint; and whether any associations more strongly drive change in cognition or mRS.

\section{Methods}

\section{Participants and design}

We prospectively recruited 264 patients presenting to stroke services in Edinburgh, UK with acute minor ischaemic stroke. All patients were dementia-free at recruitment and were treated with secondary stroke prevention according to guidelines.

Participants had baseline assessments 1-3 months post-stroke including MRI, cognitive, and mRS assessments. Participants repeated MRI at one year and repeated cognitive and mRS assessments at one and three years. Figure 1 shows variables collected at each visit.

\section{Longitudinal cognitive, mRS, and other clinical assessments}

Study procedures including baseline variables and stroke subtyping have been described in full previously., ${ }^{2,90-22}$ Participants underwent the Addenbrooke's Cognitive Examination-Revised version (ACE-R) at all three visits. The ACE-R is sensitive to multi-domain cognitive impairment post-stroke, similar to the Montreal Cognitive Assessment. ${ }^{23}$ We assessed the mRS at all three visits. We collected NIHSS at baseline and one year. At baseline, participants completed the National Adult Reading Test (NART), a durable measure of premorbid intelligence. ${ }^{22}$ 


\section{Magnetic Resonance Imaging}

\section{MRI acquisition}

Participants had brain MRI at baseline and were invited to repeat MRI one year later, all performed on the same 1.5T scanner (Signa LX; General Electric, Milwaukee). MRI included 3D T1-, T2-, and gradient recalled echo T2*-weighted, fluid-attenuated inversion recovery (FLAIR), and diffusion tensor imaging sequences using a selfshielding gradient and 8-channel phased-array head coil (details described previously). ${ }^{24}$

\section{MRI analysis}

We analysed all images according to the Standards for Reporting Vascular changes on nEuroimaging (STRIVE), ${ }^{25}$ using validated computational pipelines and visual assessments. Image analysis methods are outlined in full elsewhere ${ }^{24}$ In brief, we co-registered structural sequences at both time points using FSL-FLIRT. Total brain WMH volumes were quantified semi-automatically using paired T2-weighted, FLAIR and $\mathrm{T}^{*}$-weighted images. WMH were carefully distinguished from the site of all chronic, index, and incident stroke lesions to avoid distorting WMH volume measurements. ${ }^{26}$ This was guided by visual checks of DWI, T2-weighted and FLAIR images in discussion with a neuroradiologist (JMW). ${ }^{26}$

We extracted baseline intracranial volume (ICV) semi-automatically using the T2*weighted sequence followed by manual correction. All results were checked and manually edited independently and blinded to all clinical information.

\section{Standard Protocol Approvals, Registrations, and Patient Consents}

This study was approved by Lothian Ethics Medical Research Committee (REC 09/81101/54) and NHS Lothian R\&D Office (2009/W/NEU/14). All participants gave written informed consent. 


\section{Statistical analysis}

\section{Data preparation}

We used all available data at each time-point. To improve model fit, we transformed WMH volumes, calculating the cube root and expressing the result as \% ICV, described in previous analyses. ${ }^{2}$ We also rescaled ICV to ensure model convergence, due to the difference in magnitude vs other variables, dividing by 1000 to improve model fit. To avoid model overfitting, we calculated a baseline vascular risk factor composite sum score. ${ }^{27}$ This score assigned equal weight to hypertension, hyperlipidaemia, diabetes mellitus, and smoking history. We created WMH volume change quintiles between baseline and one year for display purposes but we used continuous WMH volume measurements for all statistical analyses. Years since stroke was categorised as 0 (baseline visit was 1-3 months post-stroke) vs 1-year visit.

\section{Models}

Figure 1 describes the cross-sectional and longitudinal data analyses.

First, we assessed whether cross-sectional WMH volume-cognition-mRS associations are stronger or weaker at the different key time-points after stroke. To achieve this, we ran linear models with ACE-R at each visit and ordinal regression models with $\mathrm{mRS}$ at each visit (Figure 1), all adjusted for key factors which we outline below.

Secondly, we assessed longitudinal change according to intra-individual trajectories. To do this, we performed mixed-effects analyses. To assess cognitive decline, we used a linear mixed-effects model (Ime4, ${ }^{28} \mathrm{R}$ ) with longitudinal change in ACE-R score between baseline and one year as the dependent variable. Since data for 
participants who did not attend follow-up visits or who died after baseline $(n=5$ at one year; $n=22$ at three years) are not missing at random, we did not perform multiple imputation or last observation carried forward in accordance with guidance ${ }^{29,30}$ and therefore visits with missing data for such participants were not used. However, where available, baseline and/or one-year data for these participants were still used in the mixed-effects models. In keeping with previous analyses of post-stroke cognitive course, ${ }^{9}$ we adjusted for normalised longitudinal WMH volumes, baseline and one-year mRS, time since stroke (baseline vs 1 year), and the following baseline variables: age, NART score, stroke subtype, vascular risk factors, and ICV (to account for original brain size) ${ }^{25}$

To assess worsening mRS, we performed mixed-effects ordinal regression analysis (mixor, ${ }^{31} \mathrm{R}$ ) with longitudinal mRS change between baseline and one year (dependent variable). In this model, we adjusted for normalised longitudinal WMH volumes (baseline to one year), baseline and one year ACE-R, NIHSS, time since stroke (baseline vs 1 year), and baseline age and stroke subtype. For participants who died after baseline or one-year follow-up, we used any available baseline or one-year data collected when these patients were alive in mixed-effects models. We did not adjust for sex since it appears not to predict long-term independence ${ }^{32}$ or cognitive function ${ }^{11}$ after stroke. For this ordinal analysis, we standardised normalised WMH volumes using Z-scores. This minimises the parameter scaling issues with ordinal dependent variables that can hinder model convergence. Finally, to assess co-varying change in cognition and mRS between baseline and one year, we created a multivariate mixed-effects model combining cognitive and mRS scores as a co-primary outcome. We fitted baseline and one-year normalised WMH volumes, NIHSS, time since stroke (baseline vs 1 year), and the following 
baseline variables: age, ICV, stroke subtype, and vascular risk factor score as fixed effects. We built the model using ASReml-R version $4 .^{33}$ This analysis included baseline and one-year data since we collected imaging data at these visits only. We excluded individual participants' time-points that did not contain a one-year WMH volume measurement $(n=68 / 264)$ or baseline vascular risk factor sum score ( $n=2 / 264)$. For all mixed-effects analyses, we fitted individual participants as random effects. The benefit of this approach is that it assesses intra-individual variation over time. We reported conditional F-test and p-values for fixed effects. We chose predictors based on previous research ${ }^{9}$ and did not perform power calculations on existing data as per recommended practice. ${ }^{34}$

\section{Data availability}

Anonymized data not published within this article can be made available by request from any qualified investigator.

\section{Results}

We collected mRS, imaging, and cognitive data at baseline ( $m R S n=264$; imaging: $n=264$; cognitive $n=157$ ), one year (mRS $n=264$; imaging: $n=196$; cognitive $n=151$ ), and three years post-stroke (mRS $n=222$; no imaging; cognitive $n=152$ ).

Participants who did not attend one-year follow-up $(n=68 / 264)$ were older (mean age 69.9 [SD 13.3] vs 65.8 [11.1]), had lower ACE-R scores (mean 83 [SD 9.7] vs 88.8 [7.6]), and had similar WMH volumes (median 13.4 [IQR 5.0-36.8] vs 12.8mL [4.433.7]) and vascular risk factor scores (mean 1.8 [SD 1.0] vs 1.6 [0.8]) at baseline than participants who did attend. Participants who did not attend three-year cognitive follow-up ( $n=112 / 264)$ were older at baseline (68.6 [SD 12.5] vs 65.6 [11.0]), had similar baseline ACE-R scores (86.9 [8.6] vs 88.7 [7.8]), higher baseline WMH 
volumes (median 17.8 [IQR 5.9-40] vs 12.3mL [3.8-29.4]), and similar vascular risk factor scores (mean 1.8 [1.0] vs 1.6 [0.94]) than those who did. Differences are shown in eTable 3 in the Supplement. At one year follow-up, 5/264 (1.9\%) had died. At three-year follow-up, 22/222 had died (9.9\%).

At baseline, the mean age was 66.9 (SD 11.8) years; $41.7 \%$ were female; median mRS was 1 (IQR 1-2); median NIHSS was 1 (IQR 0-2); median WMH volume was 13.1 $\mathrm{ml}$ (IQR 4.5-34ml); median WMH as \%ICV was 0.89 (IQR 0.31-2.38), and mean ACE-R was 88.1 (SD 8.1). Stroke subtype was lacunar in 44.6\%. Population characteristics at baseline are shown in Table 1.

Over follow-up, the mean difference in WMH volumes between baseline and one year was $1.31 \mathrm{~mL}$ (SD 8.68) with maximum $29.1 \mathrm{~mL}$ growth and maximum $31.9 \mathrm{~mL}$ shrinkage. The mean change in ACE-R scores from baseline to three years was 0.17 (SD 5.93), with a maximum increase of 20 and maximum decrease of 24 points. The median change in mRS from baseline to three years was 0 (IQR -1 to 0$)$, with a maximum increase of three and maximum decrease of four points. Figure 2 shows the distribution of change in these variables during follow-up. Figure 3 shows ACE-R and $\mathrm{mRS}$ interrelationships at each visit according to $\mathrm{WMH}$ change quintiles. See eTable 4 in the Supplement for mean WMH volumes, cognitive and mRS scores at each time-point.

Between baseline and one- or three-year follow-up, $n=95 / 195$ (48.7\%) participants had $\mathrm{a} \geq 1$-point increase in $\mathrm{mRS}$ and $76 / 148(51.3 \%)$ had $\mathrm{a} \geq 1$-point decrease in ACE-R. Considered together, $n=28 / 137$ (20.4\%) participants had a combined increase in $\mathrm{mRS}$ with decrease in ACE-R, potentially meeting DSM-V diagnostic criteria for dementia. 


\section{Multivariable analyses}

\section{Cross-sectional WMH, cognition, and mRS at baseline/one year/three years}

First, we performed cross-sectional analyses of ACE-R associations at baseline, one, and three-year visits (eTable 5 in Supplement, Figure 4A-C). At baseline, ACER scores were very weakly associated with WMH volumes (cube root as \% ICV) ( $\beta=$ 0.105, $95 \%$ confidence interval [Cl] -0.265 to 0.054 more WMH per 1-point ACE-R decrease, $p=0.195)$. At one year, associations between ACE-R scores and WMH volumes were more apparent $(\beta=-0.259,95 \% \mathrm{Cl}-0.407$ to -0.111 more $\mathrm{WMH}$ per 1point ACE-R decrease, $p=0.001$ ). The ACE-R/WMH association predominated over ACE-R associations with age, $\mathrm{mRS}$, ICV, vascular risk factors, and stroke subtype. Since participants did not undergo imaging at three years, we included normalised WMH volumes from the one-year visit in the three-year model. We did not detect any associations between one-year WMH and three-year ACE-R scores. Assessing ACE-R and mRS relationships, we did not find any association between ACE-R and mRS scores at baseline. However, we detected an ACE-R/mRS trend at one year, and a strong association emerged at three years $(\beta=-0.272,95 \% \mathrm{Cl}-0.429$ to $0.115, p=0.001$; eTable 5 in Supplement, Figure 4A-C).

We then ran cross-sectional ordinal regression models of $\mathrm{mRS}$ associations at baseline, one, and three years (eTable 6 in Supplement). We used one-year WMH volumes and NIHSS scores in the three-year model. WMH were not associated with mRS at any time-point (one-year OR 1.01, 95\% Cl 0.99-1.02). NIHSS was contemporaneously associated with $\mathrm{mRS}$ at baseline (OR 4.19, 95\% Cl 2.89-6.25), one year (OR 4.28, 95\% Cl 2.89-6.25), and three years (OR 3.08, 95\% Cl 2.517.59). 


\section{Longitudinal WMH, cognition, and mRS between baseline and one year}

Secondly, we assessed longitudinal change according to intra-individual trajectories. We used mixed-effects models to assess ACE-R, mRS and WMH volumes at baseline and one year. Changing ACE-R scores associated most strongly with age $(\beta=-0.30,95 \% \mathrm{Cl}-0.44$ to $-0.16, p<0.001)$ and NART scores $(\beta=0.53,95 \% \mathrm{Cl} 0.40-$ $0.66, \mathrm{p}<0.001)$, followed by change in normalised $\mathrm{WMH}$ volumes between baseline and one year $(\beta=-0.113,95 \% \mathrm{Cl}-0.233-0.007, p=0.065$; Figure $4 \mathrm{D}$, eTable 7$)$. ACE-R change was more strongly associated with $\mathrm{WMH}$ volume change than with mRS change, time since stroke, ICV, vascular risk factors, and stroke subtype. Change in mRS from baseline to one year was associated most strongly with NIHSS and $\mathrm{WMH}$ volumes, with a trend towards cortical (rather than lacunar) stroke subtypes (eTable 8 in the Supplement).

\section{Longitudinal WMH and combined, co-varying cognitive/mRS endpoint between}

\section{baseline and one year}

Finally, in multivariate change-change analysis, individuals with change in both ACE$\mathrm{R}$ and $\mathrm{mRS}$ between baseline and one year were more likely to have had a baselineone-year change in their WMH volumes and a baseline-one-year change in their NIHSS scores, compared with individuals without any change in ACE-R and $m R S$ (Table 2). Worsening ACE-R/mRS was also more likely in participants with lower ICV and participants who were older at baseline. Co-variation between ACE-R and mRS was stronger at the one-year than at the baseline visit.

Table 2 shows the contribution of each variable driving ACE-R change and $\mathrm{mRS}$ change between baseline and one year, e.g. change between baseline-one-year $\mathrm{WMH}$ volumes and co-varying $\mathrm{ACE}-\mathrm{R} / \mathrm{mRS}$ during the same time period is more strongly driven by WMH:mRS (standardised mRS change $=1.81$ [95\% Cl 0.35-3.26]) 
than by WMH:ACE-R (standardised ACE-R change -0.99 [95\% Cl -3.13-1.15]). In contrast, co-varying ACE-R/mRS change associations with baseline ICV and age are more strongly associated with changing ACE-R than with changing $\mathrm{mRS}$.

\section{Discussion}

We found that co-varying longitudinal change in cognition and mRS post-stroke independently associates with change in total WMH volumes between baseline and one year. In the subacute phase post-stroke, cross-sectional cognitive scores are most strongly associated with age and pre-morbid cognitive ability (NART), followed by $\mathrm{WMH}$ volume. However, by one year, the $\mathrm{WMH}-$ cognition cross-sectional association is considerably stronger and supersedes all other factors (Figure 4).

Longitudinal change in cognitive scores associates most strongly with age and NART, followed by change in WMH volumes, then mRS, baseline ICV, baseline vascular risk factors, stroke subtype, and timing (baseline vs 1 year) since stroke. A WMH-mRS association emerges over time that was not detectable on crosssectional analyses, reflecting that $\mathrm{WMH}$ and $\mathrm{mRS}$ are dynamic and effects may take longer to emerge and stabilise.

Of particular relevance to dementia after stroke, we show that longitudinal change in co-varying cognition and $\mathrm{mRS}$ in the first year post-stroke is associated with age, ICV, change in WMH volumes and NIHSS.

These findings highlight the role of SVD in evolving vascular cognitive impairment and physical dysfunction after stroke, suggesting that individuals with $\mathrm{WMH}$ progression post-stroke are more likely to develop a simultaneous decline in cognition and mRS compared to individuals with unchanging WMH after accounting 
for demographics and risk factors. This analysis builds on the recent finding in the same population that cognitive impairment after lacunar stroke is more related to background SVD than to index lesion location. ${ }^{35}$ Notwithstanding that, considering the range of all subtypes of stroke, lesion location does appear to pay a role in post stroke cognitive impairment. ${ }^{36}$ These findings add clarity to the time-course of vascular cognitive impairment and the impact of $\mathrm{WMH}$ progression in the first year after stroke, both increases and decreases, suggesting that clinical evidence of SVDrelated cognitive or mRS change becomes progressively apparent in the chronic rather than subacute phase post-stroke (Figure 3).

\section{Our research findings in context}

Our finding of associations between cognitive decline and $\mathrm{WMH}$ progression was not detected in most previous longitudinal imaging studies after stroke. ${ }^{12-14,16,17}$ These differences may be due to variation in follow-up sample sizes, cognitive measures, methods of assessing $\mathrm{WMH}$ change, lower drop-out rates, and follow-ups at the same vs separate scanners. Moreover, the interval between stroke presentation and baseline MRI assessment is an important factor (eTable 2) as SVD lesions are dynamic. ${ }^{1}$ When assessing post-stroke cognition, it is important to adjust for factors that associate with pre-morbid cognitive ability, ${ }^{22}$ later-life cerebrovascular disease ${ }^{41,42}$ and cognitive decline ${ }^{42}$ One previous study adjusted for ICV and education ${ }^{12}$ and another adjusted for premorbid $I Q .^{37}$

Apart from WMH, there are a broader range of SVD features that require further longitudinal attention in relation to combined cognitive decline ${ }^{37}$ and worsening $\mathrm{mRS}$ after stroke. Such features, including lacunes, perivascular spaces, and microbleeds are part of the total SVD picture. ${ }^{25}$ Total baseline SVD scores are associated with lower cognitive ability in older adults ${ }^{43}$ and with worse prognosis in stroke patients. ${ }^{44}$ 
Further work is required to establish how total longitudinal SVD changes might relate to clinical outcomes.

\section{Strengths and limitations}

This study uses a statistical approach combining cognition and mRS as a co-primary outcome, paralleling real-world DSM-V diagnostic criteria ${ }^{7}$ for the clinical diagnosis of dementia. We used a well-validated approach to quantify WMH volumes at two timepoints with improved retention ( $n=196 / 264)$ for follow-up MRI compared with previous longitudinal SVD studies assessing cognition after stroke ( $n=94 / 115$ at one year and $74 / 115$ at two years; ${ }^{14,37} n=52 / 101 ;{ }^{12} n=101 / 189$ [CT-based]; ${ }^{38} 52 / 81$ at five years ${ }^{17}$ ), and comparable to non-stroke longitudinal MRI studies. ${ }^{5,39,40}$

Our study had limitations. We did not invite participants to attend MRI at three years: longer-term WMH volumes would have strengthened our analysis and better reflected the dynamic changes of WMH, cognition, and $\mathrm{mRS}$ at all three time-points. Not all participants completed cognitive assessments (eTable 3 in the Supplement) because we introduced cognitive tests after the $1^{\text {st }}$ patients had joined the study but completion rates for mRS were good (84\% at three years; eTable 3). We did not include depression as a covariate: depression is common post-stroke, has associations with worse $\mathrm{WMH}$ and has implications for cognitive dysfunction and dependence.

\section{Implications for post-stroke cognitive impairment and dependence}

This study found that the key components of the dementia syndrome, combined decline in cognition and independence, are associated with the presence and progression of $\mathrm{WMH}$ in the year after a stroke. One-year, but not baseline, $\mathrm{WMH}$ volumes associate strongly with contemporaneous cognitive scores, superseding 
other clinical factors. This suggests that dynamic $\mathrm{WMH}$ in the weeks after a stroke do not reflect permanent brain damage but by one year, perhaps when any modifiable component of WMH such as interstitial oedema has cleared, ${ }^{45,46} \mathrm{WMH}$ volumes may more closely represent the underlying 'permanent' damage and hence better correlate with cognition. Studies evaluating patients for as long as possible post-stroke, i.e. to three years and beyond, are needed to capture the full long-term implications.

These results call for an acceleration of research into processes driving SVD-related cognitive decline. The emergence of a WMH-cognitive association one year after a stroke represents a short but achievable therapeutic window for halting SVD progression and potentially preserving cognitive ability and independence poststroke.

\section{Future research targets}

It would be useful to determine whether a combined, co-varying cognitive and $\mathrm{mRS}$ outcome could predict conversion to incident dementia in the longer term. We should encourage consistent analysis of levels of independence alongside cognitive ability in stroke research. A more detailed spatial analysis assessing patterns with persisting WMH vs WMH that dynamically appear and disappear would give more precise insights into the influence of SVD on the clinical course after a stroke. Moreover, an analysis of whether WMH lesions behave differently adjacent to vs remote from the stroke lesion, whether this impacts on lesion evolution, and whether this influences outcomes after stroke, is required.

Despite secondary prevention, vascular risk factors were not associated with change in cognitive scores in our study. This reinforces that finding alternative treatment approaches to target the underlying pathophysiology of SVD is particularly important. 
We need to closely track the natural history of dementia post-stroke and determine whether clinically and radiologically distinct dementia subtypes emerge over time. Identifying subgroups will allow future triage of clinical presentations to appropriate services, the development of disease-specific management strategies, and targeted entry into future research trials.

WNL-2022-200298_sup -- http://links.Iww.com/WNL/B796

\section{References}

1. Wardlaw JM, Smith C, Dichgans M. Small vessel disease: mechanisms and clinical implications. Lancet Neurol. Jul 2019;18(7):684-696. doi:10.1016/S1474-4422(19)30079-1 2. Wardlaw JM, Chappell FM, Valdes Hernandez MDC, et al. White matter hyperintensity reduction and outcomes after minor stroke. Neurology. Sep 5 2017;89(10):1003-1010. doi:10.1212/wnl.0000000000004328

3. Ramirez J, McNeely AA, Berezuk C, Gao F, Black SE. Dynamic Progression of White Matter Hyperintensities in Alzheimer's Disease and Normal Aging: Results from the Sunnybrook Dementia Study. Front Aging Neurosci. 2016;8:62. doi:10.3389/fnagi.2016.00062

4. Al-Janabi OM, Bauer CE, Goldstein LB, et al. White Matter Hyperintensity Regression: Comparison of Brain Atrophy and Cognitive Profiles with Progression and Stable Groups. Brain Sci. Jul 19 2019;9(7)doi:10.3390/brainsci9070170

5. van Leijsen EMC, van Uden IWM, Ghafoorian M, et al. Nonlinear temporal dynamics of cerebral small vessel disease: The RUN DMC study. Neurology. Oct 10 2017;89(15):1569-1577. doi:10.1212/wnl.0000000000004490

6. Raz N, Yang YQ, Rodrigue KM, Kennedy KM, Lindenberger U, Ghisletta P. White matter deterioration in 15 months: latent growth curve models in healthy adults. Neurobiol Aging. Feb 2012;33(2):429.e1-5. doi:10.1016/j.neurobiolaging.2010.11.018

7. American Psychiatric Association. Diagnostic and Statistical manual of mental Disorders. 5th edition. (DSM-5). Washington, DC: American Psychiatric Publishing; 2013. 8. Román GC, Tatemichi TK, Erkinjuntti T, et al. Vascular dementia: diagnostic criteria for research studies. Report of the NINDS-AIREN International Workshop. Neurology. Feb 1993;43(2):250-60. doi:10.1212/wnl.43.2.250

9. McHutchison CA, Cvoro V, Makin S, Chappell FM, Shuler K, Wardlaw JM. Functional, cognitive and physical outcomes 3 years after minor lacunar or cortical ischaemic stroke. Journal of Neurology, Neurosurgery \& Psychiatry. 2019;90(4):436-443. doi:10.1136/jnnp-2018-319134 
10. Georgakis MK, Duering M, Wardlaw JM, Dichgans M. WMH and long-term outcomes in ischemic stroke: A systematic review and meta-analysis. Neurology. Mar 19 2019;92(12):e1298-e1308. doi:10.1212/wnl.0000000000007142

11. Pendlebury ST, Rothwell PM. Prevalence, incidence, and factors associated with prestroke and post-stroke dementia: a systematic review and meta-analysis. The Lancet Neurology. 2009/11/01/ 2009;8(11):1006-1018. doi:https://doi.org/10.1016/S14744422(09)70236-4

12. Sachdev PS, Lipnicki DM, Crawford JD, Wen W, Brodaty H. Progression of cognitive impairment in stroke/TIA patients over 3 years. Journal of neurology, neurosurgery, and psychiatry. Dec 2014;85(12):1324-30. doi:10.1136/jnnp-2013-306776

13. Grau-Olivares M, Arboix A, Junqué C, Arenaza-Urquijo EM, Rovira M, Bartrés-Faz D. Progressive gray matter atrophy in lacunar patients with vascular mild cognitive impairment. Comparative Study

Research Support, Non-U.S. Gov't. Cerebrovasc Dis. 2010;30(2):157-66. doi: 10.1159/000316059. Epub 2010 Jun 10.

14. Lawrence AJ, Zeestraten EA, Benjamin P, et al. Longitudinal decline in structural networks predicts dementia in cerebral small vessel disease. Research Support, Non-U.S. Gov't. Neurology. May 22 2018;90(21):e1898-e1910. doi: 10.1212/WNL.0000000000005551. Epub 2018 Apr 25.

15. Lopes R, Bournonville C, Kuchcinski G, et al. Prediction of Long-term Cognitive Functions after Minor Stroke, Using Functional Connectivity. Neurology. Jan 5 2021;doi:10.1212/wnl.0000000000011452

16. Xiong Y, Wong A, Cavalieri M, et al. Prestroke Statins, Progression of White Matter Hyperintensities, and Cognitive Decline in Stroke Patients with Confluent White Matter Hyperintensities. Neurotherapeutics. 2014/07/01 2014;11(3):606-611. doi:10.1007/s13311014-0270-5

17. Appelros P, Samuelsson M, Lindell D. Lacunar Infarcts: Functional and Cognitive Outcomes at Five Years in Relation to MRI Findings. Cerebrovascular Diseases. 2005;20(1):34-40. doi:10.1159/000086202

18. Consortium. M. METACOHORTS for the study of vascular disease and its contribution to cognitive decline and neurodegeneration: An initiative of the Joint Programme for Neurodegenerative Disease Research. Alzheimer's \& dementia : the journal of the Alzheimer's Association. 2016;12(12):1235-1249. doi:10.1016/j.jalz.2016.06.004 19. Sachdev PS, Lo JW, Crawford JD, et al. STROKOG (stroke and cognition consortium): An international consortium to examine the epidemiology, diagnosis, and treatment of neurocognitive disorders in relation to cerebrovascular disease. Alzheimer's \& dementia (Amsterdam, Netherlands). 2016;7:11-23. doi:10.1016/j.dadm.2016.10.006

20. Makin SD, Doubal FN, Dennis MS, Wardlaw JM. Clinically Confirmed Stroke With Negative Diffusion-Weighted Imaging Magnetic Resonance Imaging: Longitudinal Study of Clinical Outcomes, Stroke Recurrence, and Systematic Review. Stroke. Nov

2015;46(11):3142-8. doi:10.1161/strokeaha.115.010665

21. Wardlaw JM, Doubal F, Armitage P, et al. Lacunar stroke is associated with diffuse blood-brain barrier dysfunction. Ann Neurol. Feb 2009;65(2):194-202.

doi:10.1002/ana.21549

22. McHutchison CA, Chappell FM, Makin S, Shuler K, Wardlaw JM, Cvoro V. Stability of Estimated Premorbid Cognitive Ability over Time after Minor Stroke and Its Relationship with Post-Stroke Cognitive Ability. Brain Sci. May 22

2019;9(5)doi:10.3390/brainsci9050117

23. Makin SD, Doubal FN, Quinn TJ, Bath PM, Dennis MS, Wardlaw JM. The effect of different combinations of vascular, dependency and cognitive endpoints on the sample size 
required to detect a treatment effect in trials of treatments to improve outcome after lacunar and non-lacunar ischaemic stroke. Eur Stroke J. Mar 2018;3(1):66-73.

doi:10.1177/2396987317728854

24. Valdes Hernandez Mdel C, Armitage PA, Thrippleton MJ, et al. Rationale, design and methodology of the image analysis protocol for studies of patients with cerebral small vessel disease and mild stroke. Brain Behav. Dec 2015;5(12):e00415. doi:10.1002/brb3.415

25. Wardlaw JM, Smith EE, Biessels GJ, et al. Neuroimaging standards for research into small vessel disease and its contribution to ageing and neurodegeneration. Lancet Neurol. Aug 2013;12(8):822-38. doi:10.1016/s1474-4422(13)70124-8

26. Wang X, Valdés Hernández MC, Doubal F, Chappell FM, Wardlaw JM. How much do focal infarcts distort white matter lesions and global cerebral atrophy measures?

Cerebrovasc Dis. 2012;34(5-6):336-42. doi:10.1159/000343226

27. Jochems ACC, Blair GW, Stringer MS, et al. Relationship Between Venules and Perivascular Spaces in Sporadic Small Vessel Diseases. Stroke. May 2020;51(5):1503-1506. doi:10.1161/strokeaha.120.029163

28. Bates D, Mächler M, Bolker B, Walker S. Fitting Linear Mixed-Effects Models Using lme4. Journal of Statistical Software; Vol 1, Issue 1 (2015). 10/07/ 2015;

29. Sterne JA, White IR, Carlin JB, et al. Multiple imputation for missing data in epidemiological and clinical research: potential and pitfalls. Bmj. Jun 29 2009;338:b2393. doi:10.1136/bmj.b2393

30. Lachin JM. Fallacies of last observation carried forward analyses. Clin Trials. Apr 2016;13(2):161-8. doi:10.1177/1740774515602688

31. Hedeker D, Gibbons RD. MIXOR: a computer program for mixed-effects ordinal regression analysis. Comput Methods Programs Biomed. Mar 1996;49(2):157-76.

doi:10.1016/0169-2607(96)01720-8

32. Dhamoon MS, Moon YP, Paik MC, et al. Long-term functional recovery after first ischemic stroke: the Northern Manhattan Study. Stroke. Aug 2009;40(8):2805-11.

doi:10.1161/strokeaha.109.549576

33. Butler DG CB, Gilmour AR, Thompson R. ASReml version 4. Technical report, University of Wollongong. 2018.

34. Zhang Y, Hedo R, Rivera A, Rull R, Richardson S, Tu XM. Post hoc power analysis: is it an informative and meaningful analysis? General Psychiatry. 2019;32(4):e100069. doi:10.1136/gpsych-2019-100069

35. Valdés Hernández MdC, Grimsley-Moore T, Chappell FM, et al. Post-stroke Cognition at 1 and 3 Years Is Influenced by the Location of White Matter Hyperintensities in Patients With Lacunar Stroke. Original Research. Frontiers in Neurology. 2021-March-01 2021;12(241)doi:10.3389/fneur.2021.634460

36. Weaver NA, Kuijf HJ, Aben HP, et al. Strategic infarct locations for post-stroke cognitive impairment: a pooled analysis of individual patient data from 12 acute ischaemic stroke cohorts. The Lancet Neurology. doi:10.1016/S1474-4422(21)00060-0

37. Zeestraten EA, Lawrence AJ, Lambert C, et al. Change in multimodal MRI markers predicts dementia risk in cerebral small vessel disease. Neurology. 2017;89(18):1869. doi:10.1212/WNL.0000000000004594

38. Rasquin SM, Verhey FR, Lousberg R, Lodder J. Cognitive performance after first ever stroke related to progression of vascular brain damage: a 2 year follow up CT scan study. Journal of neurology, neurosurgery, and psychiatry. Aug 2005;76(8):1075-9. doi:10.1136/jnnp.2004.055541

39. Akoudad S, Ikram MA, Koudstaal PJ, et al. Cerebral Microbleeds Are Associated with the Progression of Ischemic Vascular Lesions. Cerebrovascular Diseases.

2014;37(5):382-388. doi:10.1159/000362590 
40. Jokinen H, Gouw AA, Madureira S, et al. Incident lacunes influence cognitive decline: the LADIS study. Neurology. May 31 2011;76(22):1872-8.

doi:10.1212/WNL.0b013e31821d752f

41. Backhouse EV, McHutchison CA, Cvoro V, Shenkin SD, Wardlaw JM. Early life risk factors for cerebrovascular disease: A systematic review and meta-analysis. Neurology. Mar 7 2017;88(10):976-984. doi:10.1212/wnl.0000000000003687

42. Backhouse EV, McHutchison CA, Cvoro V, Shenkin SD, Wardlaw JM. Early Life Risk Factors for Stroke and Cognitive Impairment. Current Epidemiology Reports. 2015/09/01 2015;2(3):172-179. doi:10.1007/s40471-015-0051-7

43. Staals J, Booth T, Morris Z, et al. Total MRI load of cerebral small vessel disease and cognitive ability in older people. Neurobiology of aging. 2015;36(10):2806-2811.

doi:10.1016/j.neurobiolaging.2015.06.024

44. Appleton JP, Woodhouse LJ, Adami A, et al. Imaging markers of small vessel disease and brain frailty, and outcomes in acute stroke. Neurology. Feb 4 2020;94(5):e439-e452.

doi:10.1212/wnl.0000000000008881

45. Wardlaw JM, Makin SJ, Valdés Hernández MC, et al. Blood-brain barrier failure as a core mechanism in cerebral small vessel disease and dementia: evidence from a cohort study. Alzheimers Dement. Jun 2017;13(6):634-43. doi:10.1016/j.jalz.2016.09.006

46. Wardlaw JM, Valdes Hernandez MC, Munoz-Maniega S. What are white matter hyperintensities made of? Relevance to vascular cognitive impairment. J Am Heart Assoc. Jun 23 2015;4(6):001140. doi:10.1161/jaha.114.001140

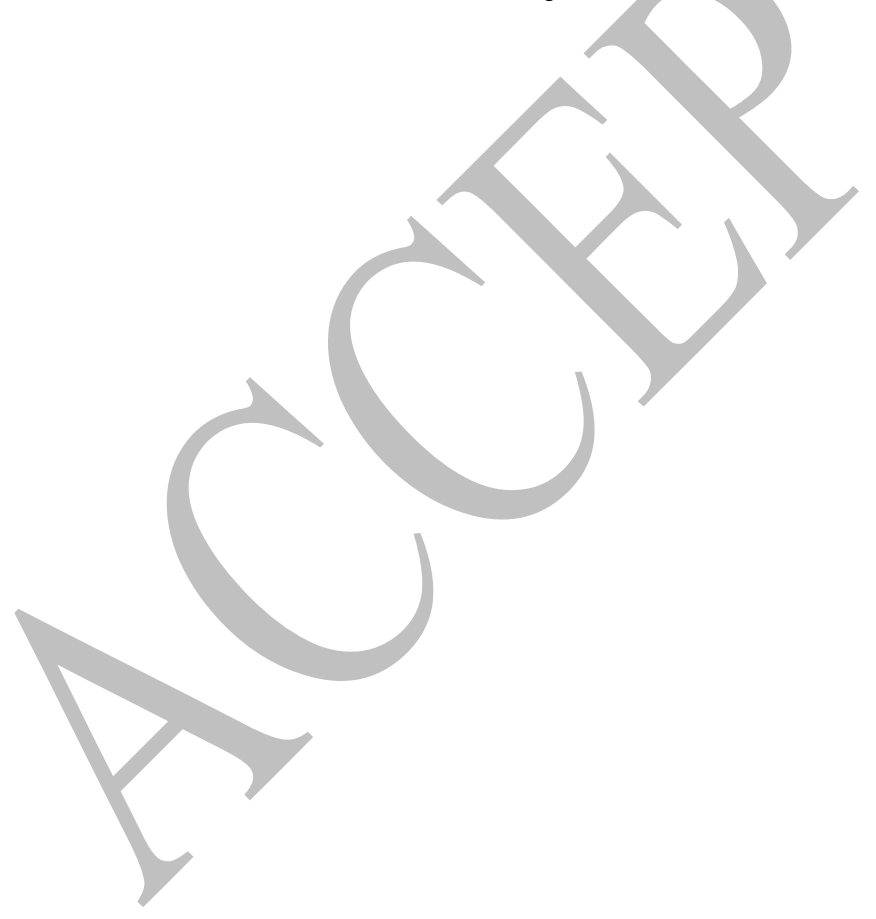




\section{Figure Legends}

\section{Figure 1 Study and analysis flow chart}

Age=age at recruitment; diabetes mellitus defined as a previous diagnosis or diagnosed at stroke presentation in accordance with the World Health Organization criteria; hypertension defined as a previous diagnosis or blood pressure of $\geq 140 / 90 \mathrm{~mm} \mathrm{Hg}$ at presentation, hyperlipidemia defined as a previous diagnosis or cholesterol $>5 \mathrm{mmol} / \mathrm{L}$ at presentation; smoking status defined as self-report of current, ex-smoker <one year ago, ex-smoker >one year ago, or never smoker; stroke subtyping defined previously, ${ }^{20}$ ICV as outlined in Methods.

ACE-R = Addenbrooke's Cognitive Assessment - Revised, mRS = modified Rankin Scale; NIHSS = National Institutes of Health Stroke Scale

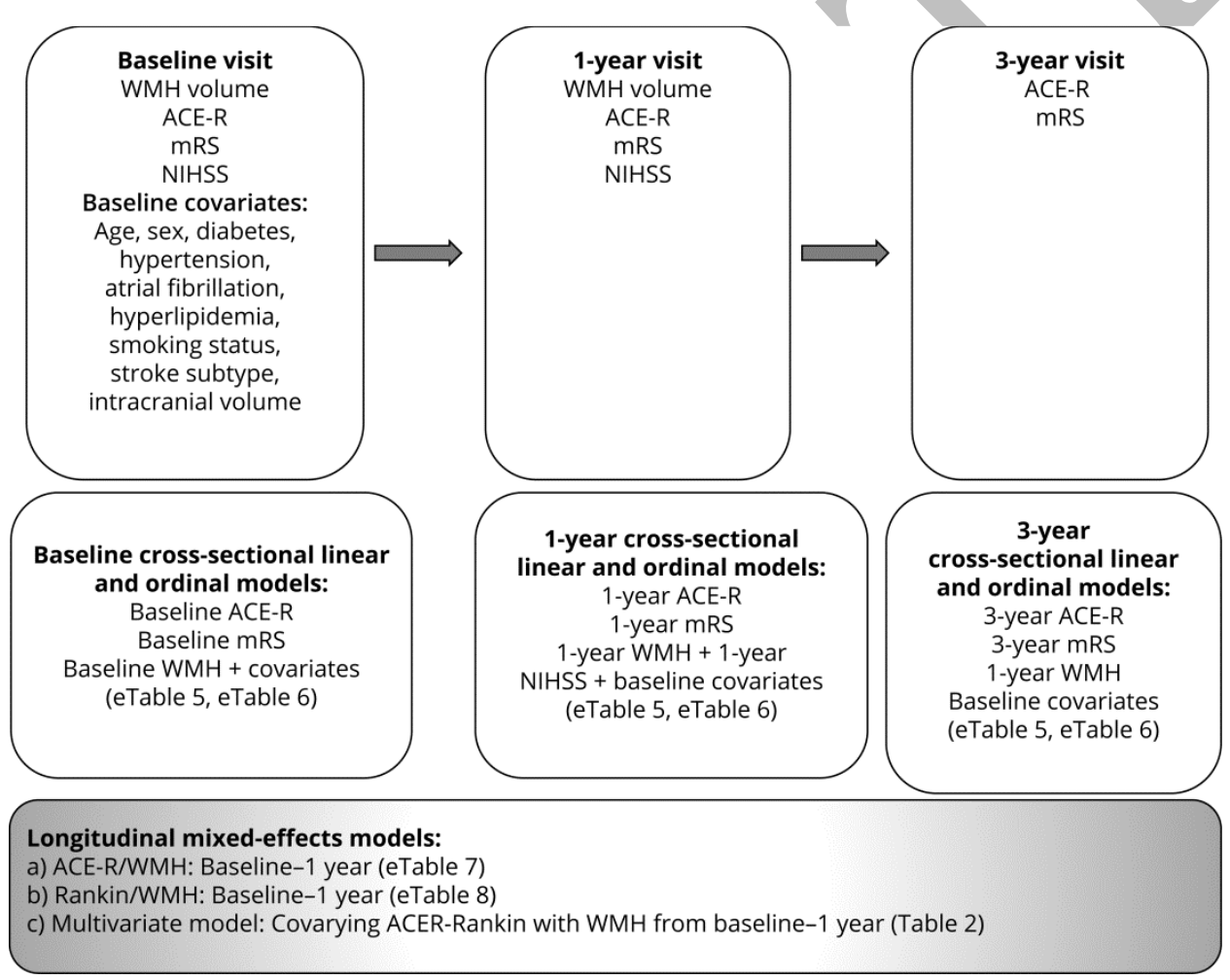


Figure 2 Distribution of change in WMH volumes (baseline-one-year), ACE-R scores and $\mathrm{mRS}$ (baseline-three-years)
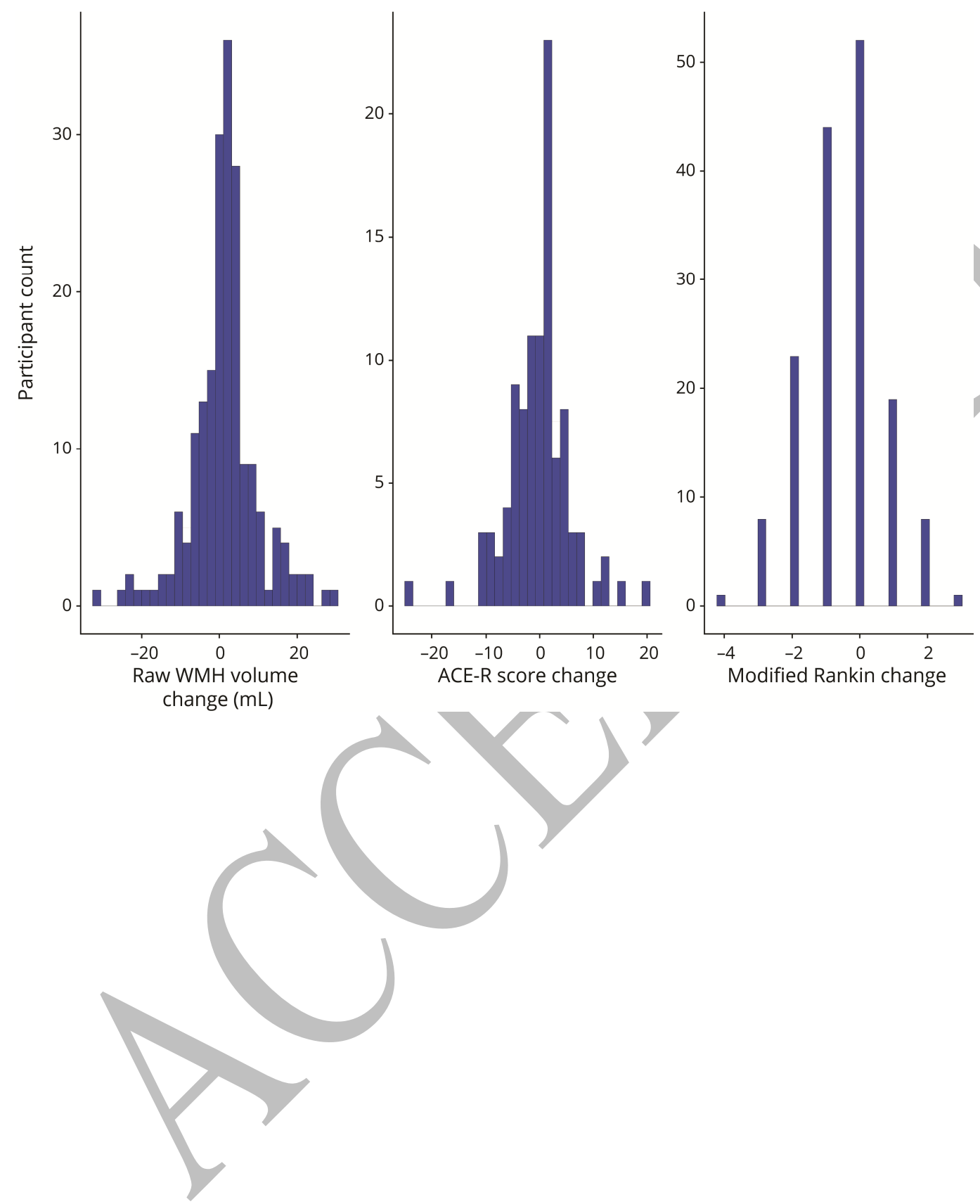
Figure 3 Cross-sectional correlations between ACE-R scores and $\mathrm{mRS}$ at each timepoint (A) baseline, (B) one-year, and (C) three-year visits, divided into panels by extent of baseline-one-year $\mathrm{WMH}$ change; $\mathrm{Q} 5=$ greatest $\mathrm{WMH}$ increase $\mathrm{Q} 1=$ greatest WMH reduction. Note that participants with $\mathrm{mRS} 5$ or 6 did not complete ACE-R

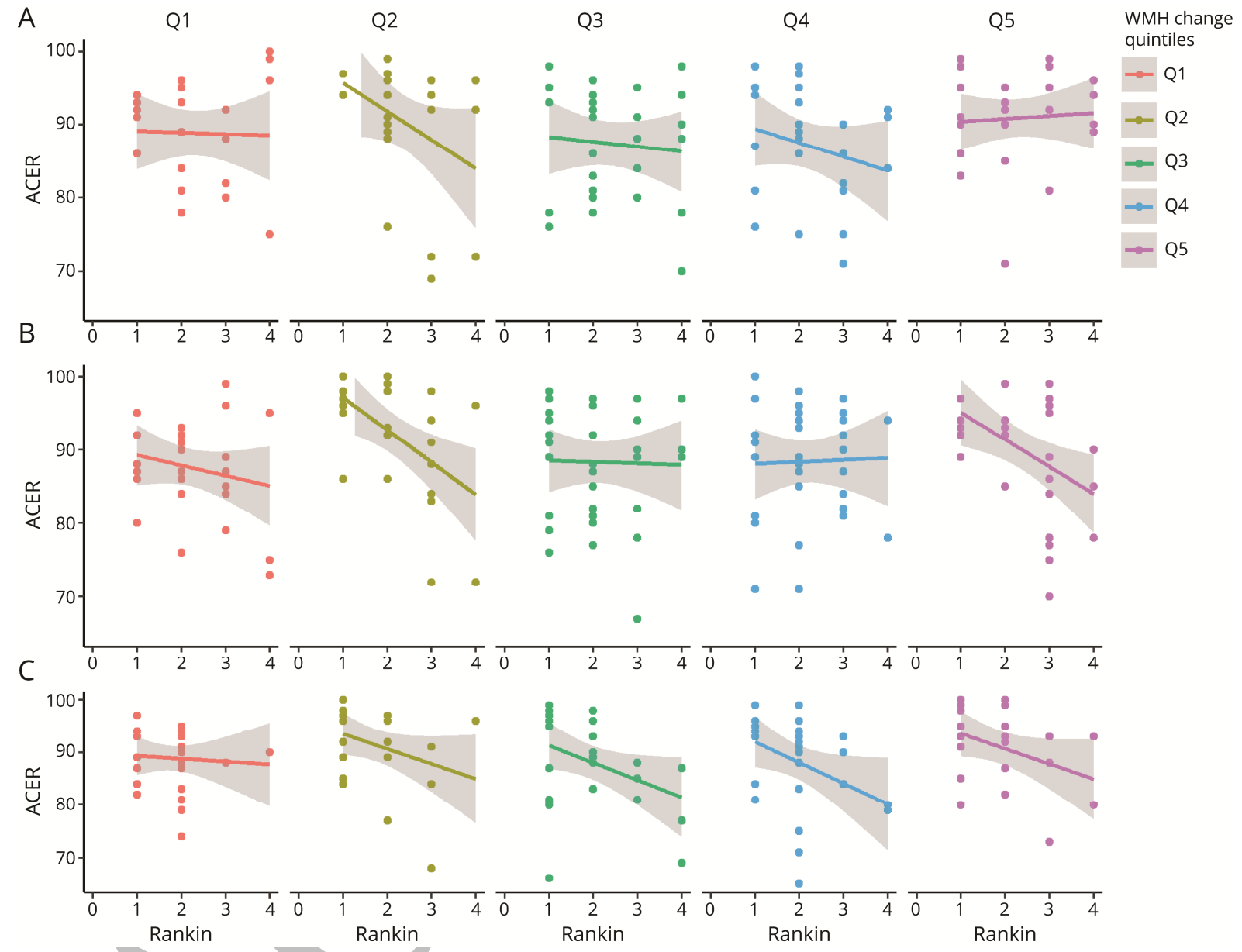


Figure 4 Cross-sectional and longitudinal associations with ACE-R scores (A-C)

Linear models: ACE-R associations at $(A)$ baseline, $(B)$ one year, $(C)$ three years, and (D) mixed-effects model: intra-individual changing ACE-R associations between baseline and one year. Note that we used one-year WMH volumes in (C)

A

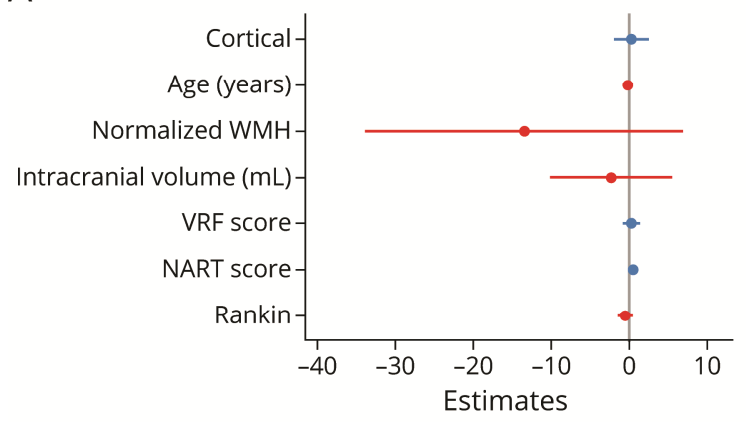

C

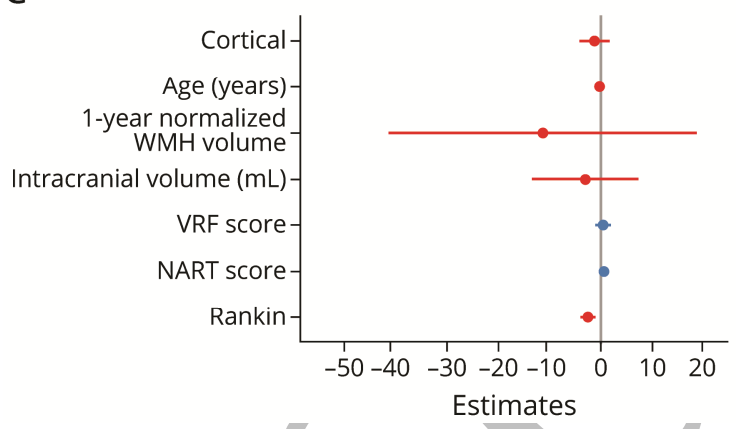

B

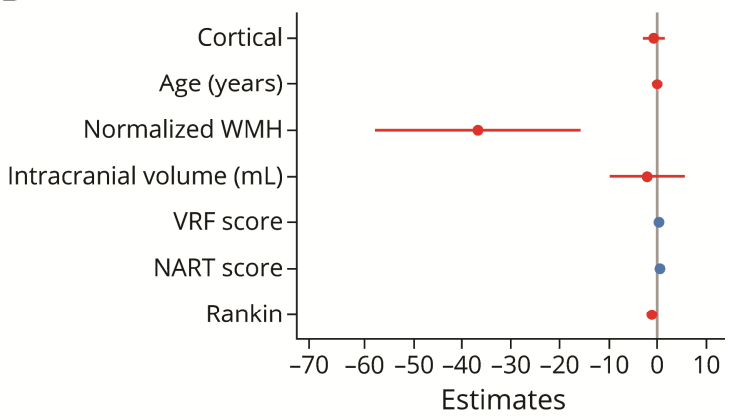

D

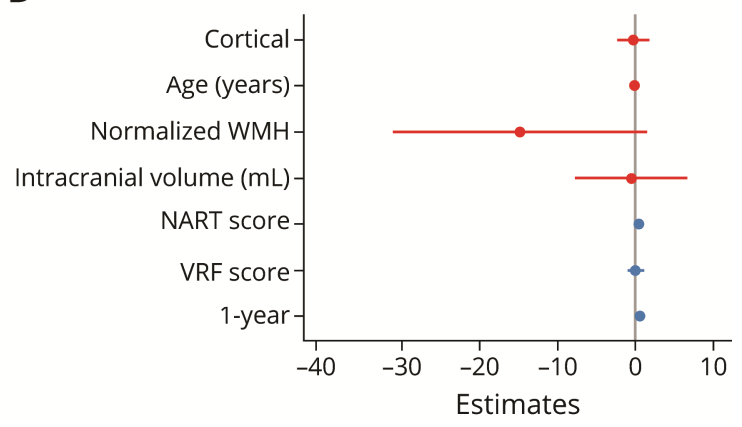




\begin{tabular}{|c|c|c|c|}
\hline & $\begin{array}{l}\text { Lacunar } \\
(\mathrm{N}=118)\end{array}$ & $\begin{array}{l}\text { Cortical } \\
(\mathrm{N}=146)\end{array}$ & $\begin{array}{l}\text { Overall } \\
(\mathrm{N}=264)\end{array}$ \\
\hline Age (years) Mean (SD) & $64.9(11.8)$ & $68.5(11.7)$ & $66.9(11.8)$ \\
\hline Female & $51(43.2 \%)$ & $59(40.4 \%)$ & $110(41.7 \%)$ \\
\hline Diabetes & $12(10.2 \%)$ & $18(12.3 \%)$ & $30(11.4 \%)$ \\
\hline Hypertension & $82(69.5 \%)$ & $109(74.7 \%)$ & $191(72.3 \%)$ \\
\hline Hyperlipidaemia & $73(61.9 \%)$ & $88(60.3 \%)$ & $161(61.0 \%)$ \\
\hline Atrial fibrillation & $7(5.9 \%)$ & $18(12.3 \%)$ & $25(9.5 \%)$ \\
\hline Ex-smoker:>one year ago & $31(26.3 \%)$ & $42(28.8 \%)$ & $73(27.7 \%)$ \\
\hline Never smoker & $35(29.7 \%)$ & $52(35.6 \%)$ & $87(33.0 \%)$ \\
\hline Ex-smoker:<one year ago & $5(4.2 \%)$ & $7(4.8 \%)$ & $12(4.5 \%)$ \\
\hline Current smoker & $46(39.0 \%)$ & $44(30.1 \%)$ & $90(34.1 \%)$ \\
\hline Vascular sum score, Mean [SD] & $1.77(0.98)$ & $1.85(0.96)$ & $1.8(0.97)$ \\
\hline NIHSS Median [IQR] & $1[0-2]$ & $1[0-1]$ & $1[0-2]$ \\
\hline mRS Median [IQR] & $1[1-2]$ & $1[1-2]$ & $1[1-2]$ \\
\hline Lacunar stroke & $118(100 \%)$ & $0(0 \%)$ & $118(44.7 \%)$ \\
\hline Cortical stroke & $0(0 \%)$ & $146(100 \%)$ & $146(55.3 \%)$ \\
\hline ACE-R score Median [IQR] & $90[82.5,94]$ & $90[83,95]$ & $90[83,94]$ \\
\hline WMH volume (ml) Median [IQR] & $13.9[4.49-35.5]$ & $12.9[4.57-33.7]$ & $13.1[4.5-34.0]$ \\
\hline WMH ml as \% ICV Median [IQR] & $0.93[0.29-2.46]$ & $0.86[0.32-2.32]$ & $0.89[0.31-2,38]$ \\
\hline
\end{tabular}


Table 2. Multivariate mixed-effects model of associations with co-varying change in ACE-R and $m R S$ scores at baseline and one year

\begin{tabular}{|c|c|c|c|c|c|c|c|}
\hline & Coefficients & $95 \% C I$ & $\begin{array}{c}\text { Scaled } \\
\text { coefficients }\end{array}$ & $\begin{array}{l}95 \% C I \\
\text { scaled }\end{array}$ & $\begin{array}{c}Z \\
\text { ratio }\end{array}$ & $\begin{array}{l}\text {-inc } \\
(A C E-\end{array}$ & $\begin{array}{l}p(A C E- \\
R: m R S)\end{array}$ \\
\hline ACE-R:Vascular risk & 0.92 & $-0.21-$ & 0.11 & $-0.03-$ & 1.56 & \multirow{3}{*}{1.6} & \multirow[b]{2}{*}{0.21} \\
\hline $\begin{array}{l}\text { factors } \\
\text { mRS:Vascular risk factors }\end{array}$ & 0.03 & $\begin{array}{r}2.05 \\
-0.04-\end{array}$ & 0.02 & $\begin{array}{l}0.25 \\
-0.06-\end{array}$ & 0.66 & & \\
\hline ACE-R:NIHSS & -0.67 & $\begin{array}{r}0.10 \\
-1.31\end{array}$ & -0.08 & $\begin{array}{l}0.10 \\
-0.16-\end{array}$ & -2.01 & & \multirow[b]{2}{*}{$<0.001$} \\
\hline mRS:NIHSS & 0.51 & $\begin{array}{l}\text { to- } 0.02 \\
0.43-\end{array}$ & 0.48 & $\begin{array}{l}0.00 \\
0.40-\end{array}$ & . & 72.0 & \\
\hline $\begin{array}{l}\text { ACE-R:ICV } \\
(I C V=\text { scaled } \div 1000)\end{array}$ & 8.76 & $\begin{array}{c}0.58 \\
0.92- \\
16.6\end{array}$ & 1.08 & $\begin{array}{l}0.55 \\
0.11- \\
2.04\end{array}$ & & 5.1 & 0.03 \\
\hline mRS:ICV & -0.48 & $-1.06-$ & -0.45 & $-1.01-$ & 1.57 & & \\
\hline $\begin{array}{l}\text { ACE-R:Normalised WMH } \\
\text { volume }(W M H=\text { cube root }\end{array}$ & -8.05 & $\begin{array}{c}n 10 \\
-25.41- \\
9.31\end{array}$ & -0.99 & & & 9.3 & 0.03 \\
\hline mRS:Normalised WMH & 1.92 & $0.39-$ & 1.81 & $0.35-$ & 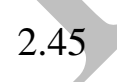 & & \\
\hline $\begin{array}{l}\text { ACE-R:Cortical subtype } \\
\text { mRS:Cortical subtype }\end{array}$ & $\begin{array}{l}-0.55 \\
0.19\end{array}$ & $\begin{array}{l}3.44 \\
-2.88- \\
1.78 \\
0.01-\end{array}$ & & $\begin{array}{l}3.26 \\
-0.35 \\
0.23 \\
0.00-\end{array}$ & $\begin{array}{r}-0.46 \\
2.08\end{array}$ & 0.0 & 0.11 \\
\hline $\begin{array}{l}\text { ACE-R:One-year visit } \\
\text { mRS:One-year visit }\end{array}$ & $\begin{array}{l}-0.06 \\
0.22\end{array}$ & 0.36 & 0.21 & $\begin{array}{c}0.33 \\
-0.11- \\
0.10 \\
0.04-\end{array}$ & $\begin{array}{l}-0.12 \\
2.59\end{array}$ & 2.2 & 0.03 \\
\hline $\begin{array}{l}\text { ACE-R:Age } \\
\text { mRS:Age }\end{array}$ & $\begin{array}{l}-0.22 \\
0.004\end{array}$ & $\begin{array}{c}0.37 \\
-0.31 \text { to } \\
0.003-\end{array}$ & $\begin{array}{l}-0.02 \\
0.0004\end{array}$ & $\begin{array}{c}0.37 \\
-0.03 \text { to } \\
n \cap n \\
-0.00-\end{array}$ & $\begin{array}{l}-4.03 \\
0.10\end{array}$ & 9.9 & $<0.001$ \\
\hline
\end{tabular}




\title{
Neurology
}

\author{
Impact of Small Vessel Disease Progression on Long-term Cognitive and Functional \\ Changes After Stroke \\ Una Clancy, Stephen DJ Makin, Caroline A McHutchison, et al. \\ Neurology published online February 7, 2022 \\ DOI 10.1212/WNL.0000000000200005
}

This information is current as of February 7, 2022

$\begin{array}{ll}\text { Updated Information \& } & \text { including high resolution figures, can be found at: } \\ \text { Services } & \text { http://n.neurology.org/content/early/2022/02/07/WNL.0000000000200 } \\ & \text { 005.full } \\ \text { This article, along with others on similar topics, appears in the } & \text { following collection(s): } \\ & \text { All Cerebrovascular disease/Stroke } \\ & \text { http://n.neurology.org/cgi/collection/all_cerebrovascular_disease_strok } \\ & \text { e } \\ & \text { All Cognitive Disorders/Dementia } \\ & \text { http://n.neurology.org/cgi/collection/all_cognitive_disorders_dementia } \\ & \text { MRI } \\ & \text { http://n.neurology.org/cgi/collection/mri } \\ & \text { Information about reproducing this article in parts (figures,tables) or in } \\ & \text { its entirety can be found online at: } \\ & \text { http://www.neurology.org/about/about_the_journal\#permissions } \\ & \text { Information about ordering reprints can be found online: } \\ \text { Permissions \& Licensing } & \text { http://n.neurology.org/subscribers/advertise }\end{array}$

Updated Information \& Services

http://n.neurology.org/subscribers/advertise

Neurology $\AA^{\circledR}$ is the official journal of the American Academy of Neurology. Published continuously since 1951, it is now a weekly with 48 issues per year. Copyright Copyright $@ 2022$ The Author(s). Published by Wolters Kluwer Health, Inc. on behalf of the American Academy of Neurology.. All rights reserved. Print ISSN: 0028-3878. Online ISSN: 1526-632X.

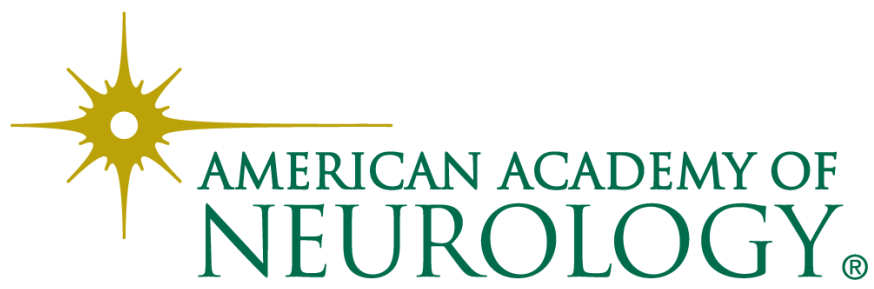

